for older and larger patients, with proper technical expertise, transfemoral intra-arterial chemotherapy can be considered in such young infants.

Disclosures S. Sur: None. B. Snelling: None. E. Peterson: None.

\section{E-032 COMBINATION OF HIGH RESOLUTION CONE-BEAM CT AND 3D DSA FOR THE EVALUATION OF INTRACRANIAL STENTS USED FOR ANEURYSM TREATMENT}

${ }^{1}$ I Yuki, ${ }^{1} \mathrm{~S}$ Hataoka, ${ }^{1} \mathrm{~T}$ Ishibashi, ${ }^{2} \mathrm{C}$ Dahmani, ${ }^{1} \mathrm{~A}$ Ikemura, ${ }^{1} \mathrm{Y}$ Kambayashi, ${ }^{1} \mathrm{I}$ Kan, ${ }^{1} \mathrm{Y}$ Abe, ${ }^{1} \mathrm{~S}$ Kaku, ${ }^{1} \mathrm{~K}$ Nishimura, ${ }^{1} \mathrm{~T}$ Kodama, ${ }^{1} \mathrm{Y}$ Sasaki, ${ }^{1} \mathrm{Y}$ Murayama. ${ }^{1}$ Neurosurgerey, The Jikei University Hospital, Tokyo, Japan; ${ }^{2}$ Siemens Japan, Tokyo, Japan

\subsection{6/neurintsurg-2016-012589.104}

Purpose Incomplete stent apposition after the treatment of brain aneurysm can increase the risk of thromboembolic complications and remains to be the major concern during the procedure. Utilizing the high resolution cone-beam CT (HR$\mathrm{CBCT}$ ) and metal artifact reduction software (MAR), the metal artifact produced by the coil mass is reduced and the visualization of the deployed stent is optimized. After combining with the $3 \mathrm{D}$ digital subtraction angiography (3D-DSA), the resulting image is used for the evaluation of the stent apposition in the artery. Initial clinical experience of this novel imaging method is reported.

Methods A total of 24 aneurysm patients who underwent the stent assisted coil embolization was selected for this study. All patients were treated using either Neuroform ${ }^{\circledR}$ stent or Enterprise ${ }^{\circledR}$ stent system. Artis PURE ${ }^{\circledR}$ Platform (Siemens) was used in this study. Acquisition protocols are follows. A HR-CBCT acquisition was performed to obtain the image of stent and coil mass. The dataset was then reconstructed using MAR. A 3 D DSA acquisition was performed for the visualization of the vasculature. The two datasets were combined using a dedicated software. A 3D volume rendering (VR) image was created and the stent apposition of each treated patient was evaluated.

Results All 24 patients underwent the image acquisition successfully. Relationship between the deployed stent and the wall of the parent artery was well visualized in every patient although partial image defect of the stent due to the metal artifact was observed in the relatively large aneurysms. The incomplete stent apposition was frequently seen near the carotid siphon, especially at the inner curve of the target vessel. Conclusion Combination of high resolution cone-beam CT and 3D DSA for the evaluation of intracranial stents provided

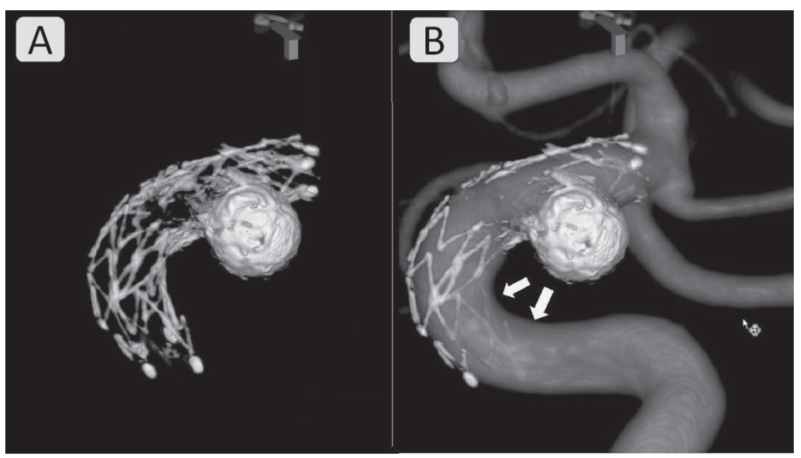

Abstract E-032 Figure 1 sufficient visualization of the deployed stent and parent artery. This imaging method can be used for the evaluation of stent apposition during/after the treatment of brain aneurysms.

Disclosures I. Yuki: 1; C; Siemens Grant. S. Hataoka: None. T. Ishibashi: 1; C; Siemens Grant. C. Dahmani: 5; C; Employee of Siemens Healthcare. A. Ikemura: None. Y. Kambayashi: None. I. Kan: None. Y. Abe: None. S. kaku: None. K. Nishimura: None. T. Kodama: None. Y. Sasaki: None. Y. Murayama: 1; C; Siemens Grant.

\section{E-033 SOFIA PLUS DISTAL ACCESS CATHETER FOR ACUTE STROKE INTERVENTION-INITIAL SINGLE CENTER EXPERIENCE}

R Cerejo, S John, A Bauer, G Toth, M Bain, M Elgabaly, T Masaryk, P Rasmussen, $\mathrm{M}$ Hussain. Cleveland Clinic Foundation, Cleveland $\mathrm{OH}$, Cleveland, $\mathrm{OH}$

\subsection{6/neurintsurg-2016-012589.105}

Introduction Endovascular thrombectomy (EVT) for acute ischemic stroke (AIS) has Level 1 evidence for improved patient outcomes compared with standard care. Effective and timely recanalization is of paramount importance to achieve this. The SOFIA Plus distal access catheter (Microvention, Tustin CA) is a newly available large lumen catheter (distal inner diameter -0.070 inch). We present our initial experience with this device for thrombectomy in acute AIS.

Methods Retrospective analysis of all patients at our center who underwent EVT for AIS using the SOFIA Plus catheter was performed. Demographic, clinical and imaging data, and early follow-up outcomes were analyzed.

Results Nine patients underwent EVT using the SOFIA Plus catheter including 6 males with a median age of 63, and a median initial NIHSS of 18. Location of the large vessel occlusion were as follows: ICA terminus - 3, M1 segment 4, M2 segment - 1, Basilar - 1. Primary direct aspiration method with the SOFIA Plus catheter was utilized in 7 cases, of which 3 required the use of a stent retriever in subsequent passes. In 2 cases, primary stent retriever plus aspiration via the SOFIA plus catheter technique was used. Median groin puncture to recanalization time was 33 minutes with median first pass to recanalization time of 9 minutes. TICI 2 b-3 recanalization was achieved in $8 / 9$ cases with a median of 2 passes. None of the patients suffered a symptomatic intracranial hemorrhage. Amongst patients in whom follow up data was available, median discharge NIHSS was 4 .

Conclusion In our single center experience, the SOFIA plus catheter was technically feasible, safe to use, and achieved high recanalization rates in a timely manner.

Disclosures R. Cerejo: None. S. John: None. A. Bauer: None. G. Toth: None. M. Bain: None. M. Elgabaly: None. T. Masaryk: None. P. Rasmussen: None. M. Hussain: None.

\section{E-034 OUTCOME PREDICTOR FOR MECHANICAL THROMBECTOMY IN ACUTE CEREBRAL ARTERY OCCLUSION}

S Park. Neurosurgery, Incheon St. Mary's Hospital, Incheon, Republic of Korea

\subsection{6/neurintsurg-2016-012589.106}

Background Acute occlusion of a major cerebral artery is associated with high mortality and morbidity. Few data about 